
\title{
28 Research Suare \\ Acute cerebral infarction with adenomyosis in a young woman: a case report
}

\section{yuan zhao ( $\nabla$ bluejayzhao@sohu.com )}

Capital Medical University Affiliated Beijing Friendship Hospital https://orcid.org/0000-0002-82120908

\section{Yongbo Zhang}

Capital Medical University Affiliated Beijing Friendship Hospital

\section{Yishu Yang}

Capital Medical University Affiliated Beijing Friendship Hospital

\section{Case report}

Keywords: Adenomyosis, acute cerebral infarction, fever, menstruation

Posted Date: April 5th, 2019

DOI: https://doi.org/10.21203/rs.2.1851/v1

License: @ (i) This work is licensed under a Creative Commons Attribution 4.0 International License. Read Full License 


\section{Abstract}

Background Acute cerebral infarction with adenomyosis in a young woman has been rarely reported. Case presentation We describe a 34 -year-old young woman who presented headache and fever $\left(38^{\circ} \mathrm{C}\right)$ for 4 days and left limb weakness for 1 day during her menstrual phase. Laboratory test data showed: Hemoglobin (HGB) (112g/L, normal: 120-150 g/L), Carcinoembryonic antigen 125 (CA125) (937.70U/ml, normal: 0-35 U/ml), D-Dimer (27.4mg/L, normal: 0-1.5mg/L). Magnetic resonance imaging (MRI) indicated acute cerebral infarction in right basal ganglia and subcortical region of right frontotemporal lobe. Further, brain computed tomography angiography (CTA) showed that the M1 segment of right middle cerebral artery was strictured and the distal branches of right middle cerebral artery were significantly less than those on the opposite side. No obvious abnormality was found in cranial magnetic resonance venogram (MRV). She had a 5-year history of adenomyosis. No tumors were found by whole body positron emission tomography-computed tomography (PET-CT). We treated this patient by using anti-infective therapy for 1 week and using anticoagulant therapy with low molecular weight heparin for 2 weeks. Subsequently, the anticoagulant therapy was discontinued and replaced by antiplatelet therapy with poliovir. We followed up this patient for 4 months, and no recurrence of cerebral infarction was found. Conclusions Acute cerebral infarction with adenomyosis may be related to elevated D-Dimer, elevated CA125, anemia, menstruation and fever. Our report suggests that acute cerebral infarction with adenomyosis can occur not only in middle-aged women but also in young women, and fever during menstrual phase in a woman with adenomyosis may be a factor leading to acute cerebral infarction.

\section{Background}

Adenomyosis is a benign uterine disease. Histopathologically, adenomyosis is characterized by the presence of ectopic endometrial tissue (endometrial glands and/or stroma) in the myometrium, surrounded by proliferation and hypertrophic smooth muscle [1]. Adenomyosis mostly occurs in women of childbearing age and mainly manifested as dysmenorrhea, menorrhea and infertility. Acute cerebral infarction with adenomyosis has been rarely reported.

Reported cases of acute cerebral infarction with adenomyosis were focused on middle-aged women over 35 years old so far. Here, we report a case of cerebral infarction in a 34-year-old young woman with adenomyosis. Besides, previous reports pay less attention to fever and menstruation of female patients with adenomyosis. In our report, acute cerebral infarction with adenomyosis was occurred in menstrual period, accompanied by fever, anemia and elevated levels of CA125 and D-Dimer. We discuss the factors leading to acute cerebral infarction with adenomyosis.

\section{Case Presentation}

A 34-year-old female patient presented headache and fever for 4 days and left limb weakness for 1 day was admitted to the hospital. Four days before admission, the patient had fever during menstruation, temperature up to $38^{\circ} \mathrm{C}$, paroxysmal headache, lower abdominal pain, muscle soreness, no dizziness, 
nausea, vomiting, intermittent cough, sputum, use of "ibuprofen". One day before admission, the patient had left limb weakness, left mouth angle askew, vague speech, no dizziness, nausea and vomiting. The patient was treated in emergency department of our hospital. She had a history of adenomyosis for 5 years, which was treated with triprillin acetate, estradiol valerate, didrogesterone, aspirin and so on. Bilateral thyroidectomy for thyroid cancer was performed 2 years before admission. At present, she takes 2 tablets of euthyrox orally every day. The patient denied hypertension, diabetes, hyperlipidemia, coronary heart disease and family history of cerebrovascular disease. The highest body temperature was $39.1^{\circ} \mathrm{C}$ after admission. Physical examination of nervous system indicated sleepiness, vague speech, left central facial-lingual paralysis, other cranial nerve examinations being normal, left limb muscle strength IV, right limb muscle strength $\mathrm{V}$, limb muscle tension being normal, limb tendon reflex symmetry. Bilateral needling sensations were normal. Left babinski's and pussep's signs were positive. Meningeal irritation sign was negative. NIHSS score was 3 points. During auscultation, the breathing sounds of both lungs were thick, and wet rales were heard.

Blood routine indicated the total number of white blood cells $\left(12.06 \times 10^{9} / \mathrm{L}\right.$, normal: $\left.3.5-9.5 \times 10^{9} / \mathrm{L}\right)$, Neutrophil percentage (84.2\%, normal: 40-75\%), HGB (112g/L, normal: 120-150 g/L). Results of admission examination showed CRP (149 mg/L, normal: 0-8 mg/L), D-Dimer (27.4 mg/L, normal: 0-1.5 $\mathrm{mg} / \mathrm{L}$ ), fibrin (-ogen) degradation products (FDP) $(69.20 \mathrm{mg} / \mathrm{L}$, normal: 0-5 mg/L), fibrinogen (Fbg) (4.79g/L, normal: 1.7-4 g/L), CA125 (937.7U/ml, normal: 0-35 U/ml), NSE (39.51ng/ml, normal: 0-18 $\mathrm{ng} / \mathrm{ml})$, CYFRA211 (3.65 ng/ml, normal: 0-3.3 ng/ml). Other tumor markers including AFP, CEA, CA199, CA153, CA724 and CA-50 were in normal range. Myocardial enzyme (CK, CK-MB, TNI, TNT) levels were in normal range. ESR, Anti chain "O", Rheumatoid factor, ANCA, ANA, ENA, Immunoglobulin and complement were in normal range. Anticardiolipin antibody, Protein $\mathrm{S}$ and protein $\mathrm{C}$ were negative. Thyroid uptake (TU), T3, T4, FT3, FT4, thyroid stimulating hormone (TSH), anti-thyroglobulin antibodies (ATG-Ab), Antithyroperoxidase antibody (ATPO) were in normal range. Sparganum mansoni-lgG antibody, Filaria antibody, Lyme-lgG antibody, Cysticercosis-lgG antibody, Leishmania spp-lgG antibody and Guangzhou roundworm-lgG antibody in blood were negative. Result of rose bengal plate test was negative. Result of tuberculosis infection T cell detection was negative. HIV antibody, HCV antibody, $\mathrm{HBsAg}, \mathrm{HBeAg}$ and Anti-HBc, Treponema pallidum antibody were negative. Cerebrospinal fluid analysis showed normal cerebrospinal fluid pressure, cell number, glucose and chloride of cerebrospinal fluid. Amphiphysin, CV2, PNMA2, Ri, Yo, Hu in cerebrospinal fluid and blood were negative. Magnetic resonance imaging (MRI) indicated acute cerebral infarction in right basal ganglia and subcortical region of right frontotemporal lobe (Figure $1 \mathrm{~A}$ and B). Further, brain computed tomography angiography (CTA) showed that the M1 segment of right middle cerebral artery was strictured and the distal branches of right middle cerebral artery were significantly less than those on the opposite side (Figure $2 \mathrm{~A}$ and B). No obvious abnormality was found in cranial magnetic resonance venogram (MRV). No tumors were found by whole body positron emission tomography-computed tomography (PET-CT). Transabdominal gynecological ultrasound suggested adenomyosis. Transthoracic echocardiography was normal. 
We treated this patient by using anti-infective therapy for 1 week and using anticoagulant therapy with low molecular weight heparin for 2 weeks. Subsequently, the anticoagulant therapy was discontinued and replaced by antiplatelet therapy with poliovir. CA125 was $202.5 \mathrm{u} / \mathrm{ml}$ on the 20th day after onset. Transvaginal Gynecological Ultrasound suggested adenomyosis on the third month after onset. We followed up this patient for 4 months, and no recurrence of cerebral infarction was found.

\section{Discussion And Conclusions}

We reported a case of a 34-year-old young woman with adenomyosis, who developed acute cerebral infarction, fever, anemia and elevated levels of CA125 and D-Dimer in menstrual period.

Regarding acute cerebral infarction with adenomyosis, we consulted cases and found 13 patients in the cases [2-8]. The median age of the 13 patients was 45.4 years. Most of the patients presented anemia and elevated levels of CA125 and D-Dimer (Table 1). 8 of the 13 patients had bilateral cerebral infarction, 1 of the 13 patients had unilateral stenosis of the right posterior cerebral artery, and 5 of the 13 patients had unilateral middle cerebral artery stenosis or occlusion. Embolus in heart was found in 2 patients (case 6, 8). 2 patients had embolism at other sites (finger in case 1, kidney in case 2). One case reported that adenomyosis can cause hypercoagulable state or diffuse intravascular coagulation (DIC), even lead to organ necrosis [9]. One 44-year-old patient of the 13 patients presented fever during her menstrual phase. Besides, one 48-year-old patient of the 13 patients also presented fever, but it was not mentioned if fever was occurred in her menstrual phase.

Many factors can cause acute cerebral infarction with adenomyosis. One of the factors is hypercoagulability, which can be indicated by elevated D-Dimer. 11 of the 13 patients showed that DDimer was elevated, and D-Dimer in our patient was also elevated. Elevated D-Dimer can indicate changes of coagulation function. D-Dimer can increase if the amount of fibrin formation is excessive or fibrinolysis is rapid and excessive [10]. Further, D-Dimer can indicate activation of coagulation and fibrinolysis system, which may lead to thrombosis or menorrhea [9].

Acute cerebral infarction with adenomyosis may be associated with elevated CA125. The elevation of CA125 was reported in 12 of the 13 patients. One serum level of CA125 in the patients even reached 2115 $\mathrm{U} / \mathrm{ml}$. CA125 is a typical mucin molecule, and the level of CA125 can be elevated in serum of patients with ovarian epithelial cancer and other tumors. Partially purified mucin can activate the coagulation system by activating factor $X$. Circulating mucus produced by cancer is considered as a susceptible factor for non-bacterial thrombotic endocarditis and disseminated thrombosis [11]. Recent experimental studies showed that carcinoma mucins promote thrombosis through the adhesion of neutrophils and platelets dependent on bidirectional signals [12].

Moreover, acute cerebral infarction with adenomyosis may be related to anemia [13]. 8 of the 13 patients suffered from anemia, and the patient in our report also suffered from anemia. If a woman with adenomyosis bleed too much during menstrual period, acute anemia can occur. Acute anemia can lead to insufficient cerebral blood flow, decreased oxygen carrying capacity, and lead to distal tissue injury. If 
hemoglobin level falls below a critical value, especially in a patient with intracranial vascular stenosis, acute anemia can have a huge negative impact on cerebral circulation, and acute anemia can lead to cerebral infarction [14].

In our report, the patient presented acute cerebral infarction with adenomyosis had fever during menstrual phase at the age of 34. In the above-mentioned cases, only one patient of the 13 patients had fever during menstrual phase, who was a 44-year-old woman. Previous cases did not mention the impact of fever during menstrual phase on patients presented acute cerebral infarction with adenomyosis, especially on young patients under the age of 35 . This time, we first pay attention to the impact of fever during menstrual phase on patients presented acute cerebral infarction with adenomyosis.

A patient with adenomyosis has normal coagulation system during non-menstrual period. During menstrual period, FDP can be increased, APTT and PT can be prolonged. These changes may be related to a pre-DIC status of a patient with adenomyosis. Disorder of coagulation system can occur during menstruation [9] [15]. Intramural hemorrhage in adenomyosis can cause blood stasis in the injured sites during menstruation, which may lead to thrombosis and subsequent microthrombosis. Microthrombosis can cause ischemic damage to the uterine myometrium. Myometrial necrosis and degradation can produce tissue factors. Blood exposed to tissue factor can activate the tissue factor coagulation pathway [9]. The pathway can cause systemic thrombin and even can cause DIC in extreme cases. Blood stagnation caused by intramural hemorrhage in adenomyosis during menstruation may activate the tissue factor (TF) coagulation pathway [3]. Although TF plays a key role in the initiation of coagulation, TF also involves in the pathogenesis of adenomyosis and may involve in angiogenesis. In addition, the increased level of TF in endometrium was associated with severe menstrual bleeding and dysmenorrhea in patients with adenomyosis. The level of CA125 can increase from premenstrual period to menstrual period. The increase of CA125 level can activate coagulation system. CA125-related infarction may occur during menstruation [8].

It is reported that fever or infection increases the incidence of acute cerebral infarction [16] [17]. Some cases report that acute respiratory and urinary tract infections can cause the increase of the incidence of stroke. The cause of stroke relates to infection load, changes in lipid metabolism, increased plasma fibrinogen, platelet activation or aggregation, platelet lysis, hypercoagulability, endothelial dysfunction, vascular smooth muscle spasm, unstable atherosclerosis and subsequent plaque rupture. Although the stimulation of inflammatory response is considered as the main cause of stroke, the mechanism of stroke caused by fever or infection is still not clearly known. Pathogens can directly invade the vascular wall during fever or infection, accompanied by proliferation of smooth muscle cell or increased production of inflammatory cytokines. In addition, regional high D-Dimer showed abnormal high levels of thrombosis and fibrin degradation products.

At present, there is no standard treatment for acute cerebral infarction with adenomyosis. It was reported that antiplatelet or anticoagulation combined gonadotropin-releasing hormone $(\mathrm{GnRH})$ agonist was used in the treatment of acute cerebral infarction with adenomyosis. However, a report showed that warfarin 
and new oral anticoagulant (NOAC) were ineffective in the treatment of cerebral infarction with adenomyosis, as the patient in the report had cerebral infarction again after having new oral anticoagulant. This report suggested that adenomyosis resection may be the most effective therapy for preventing cerebral infarction [2]. Although it was reported that benign tumor resection can improve the coagulation function of patients, reports about benign tumor resection are few [2] [18]. At present, the efficacy of surgical hysterectomy in the treatment of acute cerebral infarction with adenomyosis is rarely reported. We suggest it needs further research in clinical practices whether a patient with adenomyosis having elevated D-Dimer, elevated CA125, anemia, menstruation and fever can use anti-platelet and anticoagulant drugs in advance, in order to avoid acute cerebral infarction.

Adenomyosis is a disease occurring in women with childbearing age. At present, it is reported that causes of acute cerebral infarction with adenomyosis may be related to elevated D-Dimer, elevated CA125, anemia, menstruation and fever. The brain CTA of our 34-year-old patient suggested that M1 segment of the right middle cerebral artery was obviously narrowed. We think that the narrowed M1 segment may be related to thrombosis due to changes of coagulation function caused by adenomyosis. Acute cerebral infarction with adenomyosis can occur not only in middle-aged women but also in young women. Our report suggests that fever during menstrual phase in a female patient with adenomyosis may be a factor leading to acute cerebral infarction.

\section{Abbreviations}

\section{Ethics approval and consent to participate}

Not applicable.

\section{Consent for publication}

Written informed consent was obtained from the patient for publication of this case report and any accompanying images. A copy of the written consent is available for review by the editor of this journal.

\section{Availability of data and materials}

All data related to this case report are contained within the manuscript.

\section{Competing interests}

The authors declare that they have no competing interests.

\section{Funding}

This work was supported by the grants from National Natural Science Foundation of China (81671191 $ه$ 81371355) and Beijing Natural Science Foundation (7082028). The fundings subsidized the patient for partial examinations. 
Authors' contributions

YZ, YSY diagnosed, treated and followed up the patient, YZ drafted the first manuscript, YBZ revised the manuscript. All authors read and approved the final manuscript.

\section{Acknowledgements}

Not applicable.

\section{Authors' information}

Department of Neurology, Beijing Friendship Hospital, Capital Medical University, Beijing 100050.

\section{Endnotes}

Springer Nature remains neutral with regard to jurisdictional claims in published maps and institutional affiliations.

\section{References}

[1] Benagiano G, Brosens I, Habiba M. Adenomyosis: a life-cycle approach. Reprod Biomed Online. 2015:30:220-32.

[2] Yamashiro K, Tanaka R, Nishioka K, et al. Cerebral infarcts associated with adenomyosis among middle-aged women. J Stroke Cerebrovasc Dis. 2012: 21:910.

[3] Hijikata N, Sakamoto Y, Nito C, et al. Multiple Cerebral Infarctions in a Patient with Adenomyosis on Hormone Replacement Therapy: A Case Report. J Stroke Cerebrovasc Dis. 2016:25:e183-4.

[4] Yin X, Wu J, Song S, et al. Cerebral infarcts associated with adenomyosis: a rare risk factor for stroke in middle-aged women: a case series. BMC Neurol. 2018:18:213.

[5] Aso Y, Chikazawa R, Kimura Y, et al. "Recurrent multiple cerebral infarctions related to the progression of adenomyosis: a case report". BMC Neurol. 2018:18:119.

[6] Uchino K, Shimizu T, Mizukami H, et al. Nonbacterial Thrombotic Endocarditis Complicated by Cerebral Infarction in a Patient with Adenomyosis with High Serum CA125 Level; A Case Report. J Stroke Cerebrovasc Dis. 2018:27:e42-e45.

[7] Okazaki K, Oka F, Ishihara H, et al. Cerebral infarction associated with benign mucin-producing adenomyosis: report of two cases. BMC Neurol. 2018 :18:166.

[8] Yin X, Wu J, Song S, et al. Cerebral infarcts associated with adenomyosis: a rare risk factor for stroke in middle-aged women: a case series. BMC Neurol. 2018 :18:213. 
[9] Nakamura Y, Kawamura N, Ishiko O, et al. Acute disseminated intravascular coagulation developed during menstruation in an adenomyosis patient. Arch Gynecol Obstet. 2002:267:110-2.

[10] Yamanaka A, Kimura F, Yoshida T, et al. Dysfunctional coagulation and fibrinolysis systems due to adenomyosis is a possible cause of thrombosis and menorrhagia. Eur J Obstet Gynecol Reprod Biol. 2016:204:99-103.

[11] Yamashiro K, Furuya T, Noda K, et al. Cerebral infarction developing in a patient without cancer with a markedly elevated level of mucinous tumor marker. J Stroke Cerebrovasc Dis. 2012: 21:619.

[12] Shao B, Wahrenbrock MG, Yao L, et al. Carcinoma mucins trigger reciprocal activation of platelets and neutrophils in a murine model of Trousseau syndrome. Blood. 2011:118:4015-23.

[13] Akaishi $T$, Kuroda $H$, Tateyama $M$, et al. Recurrent cerebral infarction synchronous with menorrhagia caused by endometrial stromal sarcoma. J Neurol Sci. 2015:358:509-11.

[14] Tsai CF, Yip PK, Chen CC, et al. Cerebral infarction in acute anemia. J Neurol. 2010:257:2044-51.

[15] Son J, Lee DW, Seong EY, et al.Acute kidney injury due to menstruation-related disseminated intravascular coagulation in an adenomyosis patient: a case report. J Korean Med Sci. 2010:25:1372-4.

[16] Shan K, Guo W. Stroke caused by an inflammatory thrombus: a case report.BMC Neurol. 2017:17:33.

[17] Chen JJ, Chang HF, Liu CY, et al. Infectious Mononucleosis Complicated with Acute Cerebral Infarction: A Case Report. Acta Neurol Taiwan. 2015:24:25-9.

[18] Yoo HJ, Chang DS, Lee KH. Acute renal failure induced by disseminated intravascular coagulopathy in a patient with adenomyosis. J Obstet Gynaecol Res. 2012:38:593-6.

\section{Tables}

Table 1 summary of cases of acute cerebral infarction with adenomyosis 


\begin{tabular}{|c|c|c|c|c|c|c|c|c|}
\hline Case & Age & $\begin{array}{l}\text { CA125 } \\
(\mathrm{U} / \mathrm{ml}) \\
\end{array}$ & $\begin{array}{l}\text { D-dimer } \\
(\mu \mathrm{g} / \mathrm{ml})\end{array}$ & $\begin{array}{l}\text { HGB } \\
(\mathrm{g} / \mathrm{dl})\end{array}$ & Fever & $\begin{array}{l}\text { Menstrual } \\
\text { phase }\end{array}$ & $\begin{array}{l}\text { CI(Unilateral or } \\
\text { bilateral) }\end{array}$ & $\begin{array}{l}\text { Cerebrovascular } \\
\text { involvement }\end{array}$ \\
\hline $1[2]$ & 45 & 159 & 1.1 & 8.4 & - & no & bilateral & normal \\
\hline $2[2]$ & 44 & & - & 7 & - & & Unilateral & normal \\
\hline $3[2]$ & 50 & 42.6 & $0.57^{\mathrm{a}}$ & 6.9 & - & yes & Unilateral & normal \\
\hline $4[2]^{b}$ & 42 & 1750 & 6 & 8.6 & - & yes & bilateral & normal \\
\hline $5[3]^{\mathrm{c}}$ & 59 & 334.8 & 7 & - & - & - & bilateral & - \\
\hline $6[18]^{d}$ & 49 & 379 & 3.99 & 9.9 & no & no & bilateral & normal \\
\hline $7[4]^{b}$ & 44 & 2115 & 17 & 10.3 & $\begin{array}{l}\text { yes } \\
\left(37.7^{\circ} \mathrm{C}\right)\end{array}$ & yes & bilateral & severe stenosis in MCA \\
\hline $8[5]^{d}$ & 48 & 901 & 1.9 & 8.5 & $\begin{array}{l}\text { yes } \\
\left(37.4^{\circ} \mathrm{C}\right)\end{array}$ & - & bilateral & $\begin{array}{l}\text { occlusion of the left M1 } \\
\text { of MCA }\end{array}$ \\
\hline $9[6]$ & 42 & 395 & 1.4 & - & - & - & Unilateral & $\begin{array}{l}\text { occlusion of the left M2 } \\
\text { of MCA }\end{array}$ \\
\hline $10[6]$ & 50 & 143 & 3.7 & - & - & - & Unilateral & $\begin{array}{l}\text { occlusion of the left M1 } \\
\text { of MCA }\end{array}$ \\
\hline $11[7]$ & 34 & 937.1 & 1.050 & $134^{\mathrm{a}}$ & - & yes & bilateral & normal \\
\hline $12[7]$ & 37 & 735.7 & 2.34 & 108 & - & yes & Unilateral & normal \\
\hline 13[7] & 46 & 546.5 & 12.04 & $121^{\mathrm{a}}$ & - & yes & bilateral & stenosis of the right PCA \\
\hline 14 & 34 & 937.7 & 27.4 & 112 & yes & yes & Unilateral & $\begin{array}{l}\text { severe stenosis in the } \\
\text { right M1 of MCA }\end{array}$ \\
\hline
\end{tabular}

- indicates not mention; ${ }^{a}$ indicates normal; ${ }^{b}$ indicates the patient suffers from recurrence of acute cerebral infarction. ${ }^{\mathrm{c}}$ indicates the patient suffers from thrombus formation on the aortic valve. ${ }^{\mathrm{d}}$ indicates the patient suffers from nonbacterial thrombotic endocarditis.

\section{Figures}

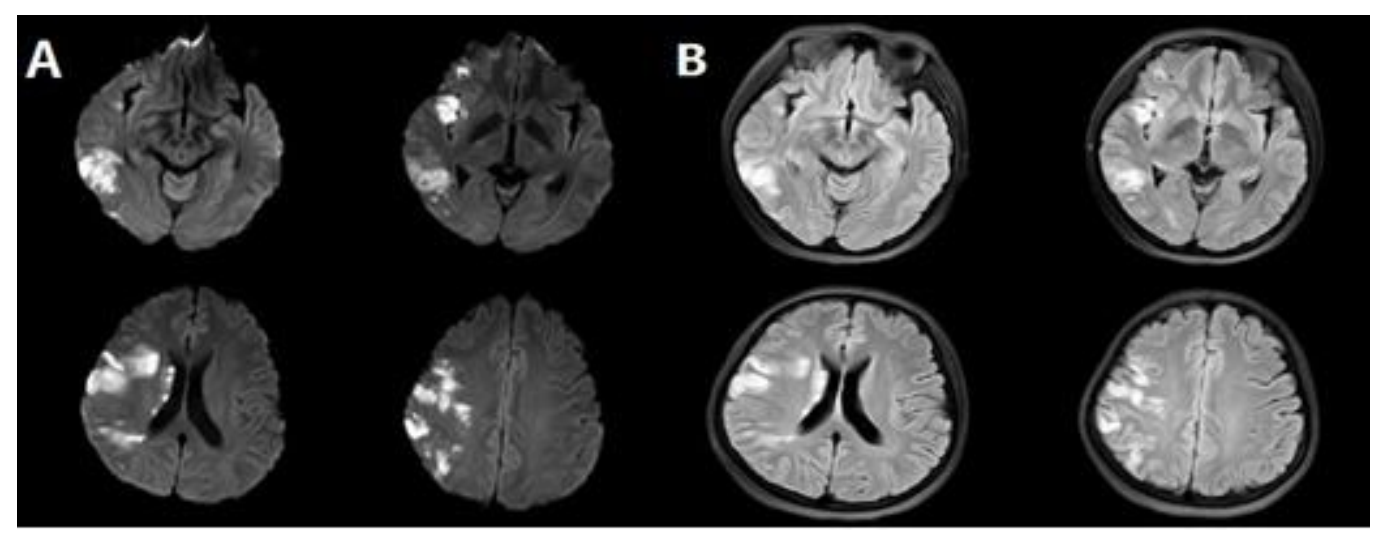

\section{Figure 1}

The MRI after two days of onset. Acute cerebral infarction in subcortical of right frontotemporal lobe and basal ganglia. (A) Diffusion weighted imaging (DWI). (B) Fluid-attenuated inversion recovery (FLAIR). 


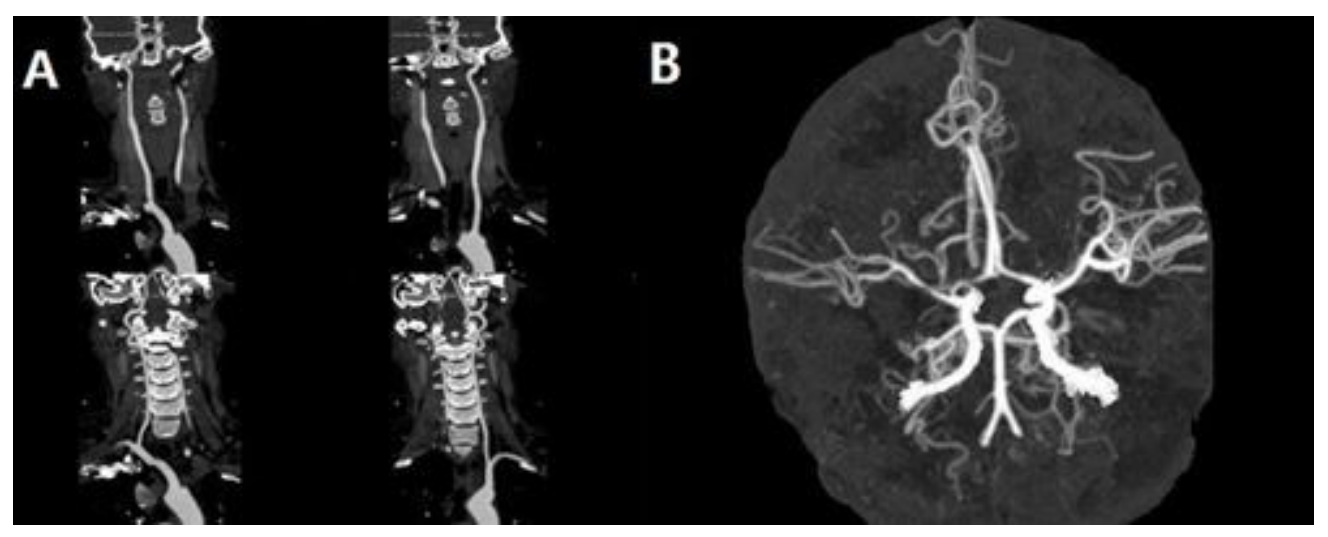

\section{Figure 2}

Brain CTA after six days of onset. (A) No abnormal carotid artery was found. (B)The M1 segment of the right middle cerebral artery was strictured and the distal branches were significantly less than those of the opposite side.

\section{Supplementary Files}

This is a list of supplementary files associated with this preprint. Click to download.

- Checklist.docx 